\title{
Integration von hochauflösenden Fernerkundungs- und Geländemodelldaten für die Landschaftsvisualisierung
}

\begin{abstract}
This paper presents experiments for realistic landscape visualization using high resolution digital aerial photographs and elevation models. The natural environment of a river in northern Switzerland is visualized. Photogrammetrically measured digital elevation data and ortho-rectified remote sensing imagery (Landsat Thematic Mapper/TM and aerial photographs) are combined to compute realistic 3D views of the landscape. To render the landscape more realistically, the digital elevation model (DEM) is transformed to a digital surface model (DSM), representing the surface and including objects like forests or bushes. For this transformation land cover information and GIS tools were used. Unnaturally looking vertical borders between different land cover classes were suppressed by interpolating transition zones. Additionally, 3D objects (trees) are used in the foreground to increase the realism of the views.
\end{abstract}

\section{Einleitung}

Das Amt für Gewässerschutz und Wasserbau des Kantons Zürich (AGW) beschäftigt sich seit mehr als zehn Jahren mit der Renaturierung von Gewässern. Im vorliegenden Projekt des Tößlaufes zwischen Sennhof und Winterthur werden mögliche Schritte zur Wiederherstellung einer natürlichen Flußlandschaft überprüft. Innerhalb dieses Projektes erhielt die Gruppe für Landschaftsvisualisierung der Remote Sensing Laboratories (RSL) der Universität Zürich die Aufgabe, von möglichen Renaturierungsschritten betroffene Abschnitte des Flußlaufes zu visualisieren. Sowohl die derzeitige Situation als auch Szenarien sollen anhand möglichst realistisch wirkender, perspektivischer Darstellungen aufgezeigt werden.

\section{Datenbeschreibung und -vorbereitung}

\section{Bilddaten}

Um von den einander ergänzenden Eigenschaften verschiedener Datensätze Gebrauch zu machen, kombinieren wir Bilddaten mit unterschiedlichen geometrischen Auflösungen. Für den Hintergrundbereich der perspektivischen Darstellungen werden Landsat Thematic Mapper-(TM-)Daten verwendet. TM-Daten werden in sieben Spektralbereichen aufgenommen. Wir benützen die drei Bänder im sichtbaren Bereich des elektromagnetischen Spektrums, die eine geometrische Auflösung von $30 \mathrm{~m}$ aufweisen. Diese grobe geometrische Auflösung erlaubt die Abdeckung einer ausgedehnten Umgebung des Untersuchungsgebietes mit Bilddaten. Die Daten entnehmen wir dem von SUTER erstellten TM-Mosaik der Schweiz (SUTER 1992). Die verwendeten TM-Daten wurden im September 1984 aufgenommen.

Aufgrund der geringen Flughöhe über Grund während der Aufnahme weisen die zur Verfügung stehenden Luftbilder eine hohe geometrische Auflösung von $50 \mathrm{~cm}$ für die Umgebung des Untersuchungsgebietes und von $10 \mathrm{~cm}$ für die speziell interessierende Talsohle auf. Die von den Luftbildern abgedeckte Fläche umfaßt etwa $30 \mathrm{~km}^{2}$ mit einer Auflösung von $50 \mathrm{~cm}$ und $10 \mathrm{~km}^{2}$ mit einer Auflösung von $10 \mathrm{~cm}$. Die Luftbildaufnahmen wurden am 5. April 1995 von der Swissair Photo + Vermessungen AG durchgeführt und umfassen das gesamte Untersuchungsgebiet. Die digitalen Luftbilddaten beanspruchen einen Speicherplatz von rund 3,5 Gigabyte.

\section{Bildverarbeitungsschritte}

Die von den verschiedenen Aufnahmesystemen herrührenden unterschiedlichen Charakteristika der Bilddaten erfordern radiometrische und geometrische Vorverarbeitungsschritte. Die Vorverarbeitungsschritte für die verwendeten TM-Daten wurden von SUTER eingehend behandelt (SUTER 1992).

\section{Geokodierung}

Zur Erstellung perspektivischer Ansichten mit Geländemodellen und Bilddaten müssen beide Datentypen im selben geometrischen Referenzsystem vorliegen. Alle verwendeten Bilddaten wurden vorgängig geokodiert. Dabei werden die durch Plattformbewegungen während der Aufnahme, durch die Abbildungsgeometrie des Sensors und durch Höhenunterschiede im Gelände auftretenden Störungen und Verfälschungen behoben.

Die analogen Luftbilder (perspektivische Zentralprojektion) wurden ihrer photogrammetrischen Auflösung entsprechend gescannt und anschließend mit Hilfe eines

Peter Pirchl, Philipp Hirtz, Martin Suter, dipl. Geogr., Daniel Nüesch, Prof. Dr., Geographisches Institut der Universität Zürich, Winterthurerstraße 190, 8057 Zürich (ppirchl,hirtz,suter, nuesch)@geo.unizh.ch 
digitalen Geländemodells zu Orthophotos (senkrechte Parallelprojektion) umgewandelt. Von den unterschiedlichen Geländehöhen abhängige Parallaxen werden durch dieses Verfahren eliminiert, so daß das resultierende Bild keine terrainbedingten Verzerrungen mehr enthält. Die einzelnen Orthophotos wurden zu einem Mosaik gruppiert, welches das Untersuchungsgebiet abdeckt.

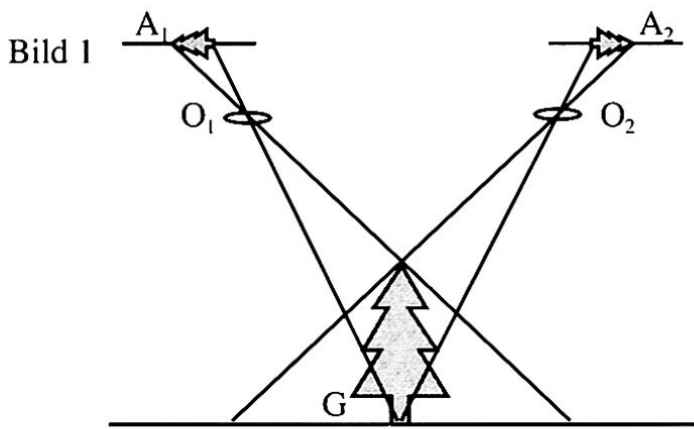

Bild 2

$A_{1}, A_{2}=$ radial verzerrte Abbilder $\quad G=$ Geländesituation $\mathrm{O}_{1}, \mathrm{O}_{2}=$ Projektionszentren

Abb.1 Radialdeformation aufgrund zentralperspektivischer Aufnahmecharakteristika (schematisch).

Die Deformation von Objekten durch die zentralperspektivischen Aufnahmecharakteristika photographischer Kameras (vgl. Abbildung 1) ist in den digitalen Orthophotos sehr gut erkennbar. Sie wird durch die geringe Flughöhe verstärkt. Objekte auf dem Luftbild, die nicht durch das Geländemodell repräsentiert sind, werden durch den Prozeß der Orthorektifizierung nicht entzerrt. Dementsprechend ergeben sich im Übergangsbereich von Bildern störend wirkende Unterschiede in der Ausrichtung der radial deformierten Objekte.

\section{Radiometrische Korrektur der Bilddaten}

Die Kombination der TM-Kanäle 3-2-1 in einem RGBFarbkomposit erzeugt Bilder mit natürlich wirkenden Farbcharakteristika. GRAF et al. beschreiben mögliche Schritte zur Farbverbesserung von TM-Szenen (GRAF et al. 1994). Innerhalb der jeweiligen Auflösung sind die Farbcharakteristika der Bilder weitgehend homogen. Der direkte Vergleich TM-, 50-cm- und 10-cm-Daten hingegen macht die Notwendigkeit einer Farbangleichung deutlich. Ein wirklich befriedigendes Resultat mit farblich unauffälligen Übergangszonen zwischen den unterschiedlichen Auflösungen konnte nicht ganz erreicht werden. Die Farbcharakteristika der 10-cm- und der 50cm-Daten, obschon am gleichen Tag, innerhalb einer Stunde und unter vergleichbaren Beleuchtungsbedingungen aufgenommen, sind bemerkenswert unterschiedlich. Die Verarbeitung der Filme war offenbar nicht dieselbe. Die Unterschiede der Farbcharakteristik zwischen
Luftbild- und Satellitendaten überraschen nicht. Erstens ist die geometrische Auflösung der Satellitendaten 60- bzw. 300mal geringer als die der Luftbilddaten, zweitens wurden die Aufnahmen zu phänologisch völlig unterschiedlichen Zeitpunkten gemacht. In den Luftbildern tragen die Laubbäume noch kein Laub. Vor allem in den am höchsten aufgelösten Bildern sieht man anstelle der Laubdecke mehrheitlich Waldboden mit gut erkennbaren Baumstämmen und Schatten derselben. Im Gegensatz dazu weisen die Wälder in den Satellitendaten geschlossene Vegetationsdecken auf. Im Übergangsbereich von Luftbildern zu TM-Szenen führt dies, speziell in bewaldeten Gebieten, zu gut erkennbaren Veränderungen der Bildinformation.

\section{Höhendaten}

Es werden Höhendaten unterschiedlicher Auflösung verwendet. Das ausgedehnte Gebiet rund um das Untersuchungsgebiet wird durch grob auflösende Geländemodelldaten repräsentiert. Der Talboden des Untersuchungsgebietes wird durch photogrammetrisch erhobene Elevationswerte abgedeckt. Geländediskontinuitäten erfassende Bruchkanten (z. B. Flußufer, Wegrand), Isolinien mit einer Äquidistanz von $0,5 \mathrm{~m}$ und unzählige Höhenkoten mit zentimetergenauer Höheninformation sind die Grundlage für eine Triangulation $\mathrm{zu}$ einem Triangulated Irregular Network (TIN).

Das geographische Informationssystem ARC/INFO stellt ein mächtiges Umfeld zur Erzeugung von TINs bereit. Das Konzept erlaubt die benutzergesteuerte Definition verschiedener Eigenschaften. Bruchkanten, Ausschnittpolygone (z. B. Inseln, interessierende Gebiete) oder Aussparungsflächen (z. B. Seen, Gebiete mit ungenügenden Daten) können festgelegt werden. Dadurch wird die Geländerepräsentation verbessert. Unterschiedliche Datentypen (Vektoren, Punktmessungen, Rasterdaten) können verwendet werden. Unglücklicherweise kann man mit einem TIN nur sogenannte funktionale Oberflächen erzeugen. Pro (x,y)-Wert eines Punktes ist höchstens ein ( $\mathrm{z})$-Wert möglich $(\mathrm{z}=f[\mathrm{x}, \mathrm{y}])$.

3-D-Objekte können daher mit TIN nur als 2,5-D-Objekte angenähert werden. Die Darstellung vertikaler oder sogar überhängender Geländepartien ist nicht möglich, da mehrere ( $\mathrm{z}$ )-Werte einem $(\mathrm{x}, \mathrm{y})$-Tupel zugewiesen werden müßten. Vertikale Elemente des Geländemodells können aber durch einen geringen Versatz des Fußpunktes vom Kopfpunkt angenähert werden.

\section{Datenmodellierung}

\section{Umwandlung eines digitalen Geländemodells (DGM) in ein digitales Oberflächenmodell (DOM)}

Zur Erstellung von Landschaftsdarstellungen wird ein Geländemodell benötigt. Das Geländemodell berücksichtigt jedoch nicht die Höhe von Objekten im Gelände 
(Gebäude, Vegetation). Speziell bei hohen geometrischen Auflösungen führt die Kombination von Geländemodell- und Bilddaten $\mathrm{zu}$ unbefriedigenden, unrealistisch wirkenden Resultaten. Zum Beispiel sollte der Blick von einer Waldlichtung aus durch den umgebenden Wald eingeschränkt sein. Bei Verwendung eines Geländemodells bildet der umliegende Wald aber kein Sichthindernis.

Die jeweilige Landnutzung muß also einen Einfluß auf die im Höhenmodell repräsentierten Werte haben, und zwar derartig, daß das Geländemodell bei Bedarf um einen der Landnutzungshöhe entsprechenden Betrag angehoben wird.

Kartographische Generalisierung und die zeitlich verschobene Datenerfassung führen zu Lagefehlern, welche Karten oder Pläne als exakte geometrische Informationsquellen für die Lage der Landnutzungspolygone unzulässig machen. Daher wurden die Luftbilder und die in den Geländerohdaten festgehaltenen Geländediskontinuitäten (Bruchkanten, photogrammetrisch aus einem Stereo-Luftbildpaar erhoben) zur Erstellung geometrisch korrekt liegender Landnutzungspolygone verwendet. Elf Landnutzungsklassen wurden erhoben und mit empirisch ermittelten Höhenwerten versehen. Diese reichen von keiner zusätzlichen Erhöhung (Wasserflächen) bis zu einer Anhebung um $18 \mathrm{~m}$ (Waldflächen). Diese neu festgelegten Höheninformationen wurden gemeinsam mit den obengenannten Höheninformationen trianguliert und ergaben ein DOM, eine Repräsentation der Oberfläche des Gebietes.

Das DOM im TIN-Format wurde in ein für die Perspektivenberechnung nötiges Rasterformat umgewandelt. Da die Beschaffenheit der Waldoberfläche weniger regelmäBig und vor allem rauher ist als die Bodenoberfläche, applizieren wir eine unregelmäßige Modulation auf die Waldflächen. Diese Modulation kann entweder, wie im von GRAF vorgeschlagenen Ansatz, aus dem Grünkanal der Luftbilder extrahiert werden (GRAF 1995) oder aber mit einer fraktalen Prozedur gerechnet werden. Sie trägt vor allem dazu bei, daß die Oberkanten der Waldränder, besonders bei flachem Blickwinkel des Betrachters, nicht scharfkantig wirken (vgl. Abbildung 2).
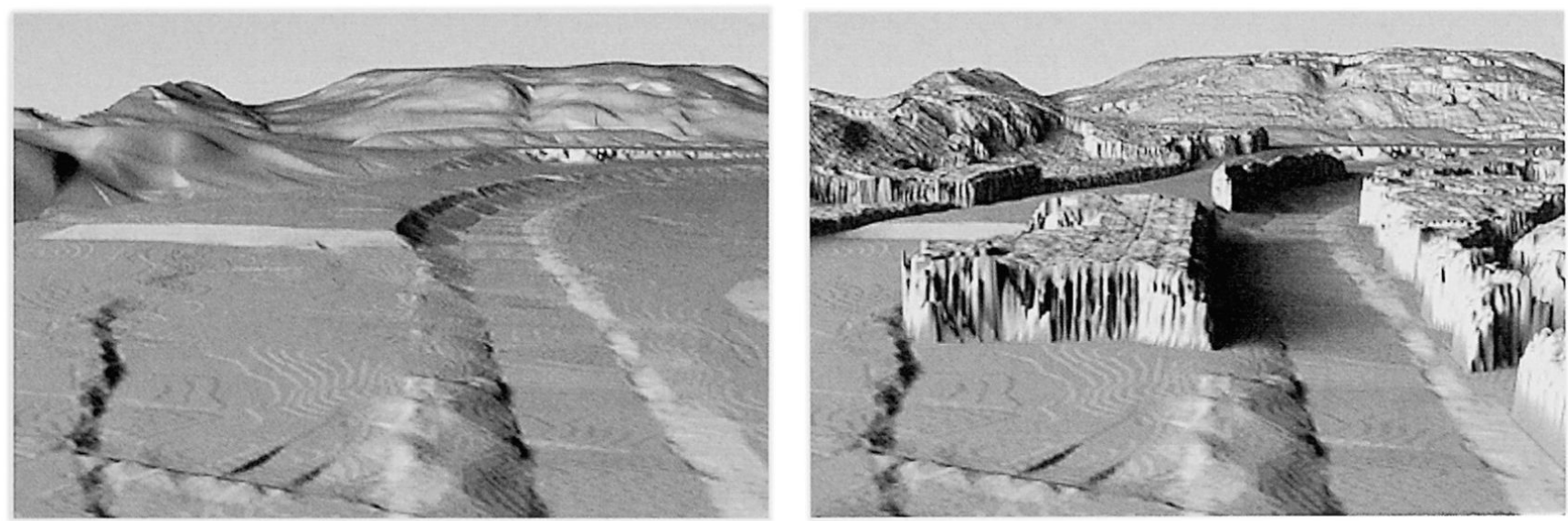

Da die Anhebung der Waldgebiete im DOM zu unschönen, senkrechten Wänden führt, schlagen wir eine zusätzliche Modulation der Waldränder vor. Dabei wird die Höhenverschiebung des DOM nicht zwischen zwei Pixeln vollzogen, sondern auf eine Pufferzone verteilt. Diese Pufferzone ist so modelliert, daß die Höhenwerte monoton, aber mit zufälligen Veränderungsbeträgen angepaßt werden. Um eine naturnahe unregelmäßige Modulation entlang des Waldrandes zu erreichen, wird die Verteilung des Anhebungsbetrages auf die Pixel innerhalb des Puffers für jede Kolonne bzw. Spalte des DOM zufällig neu generiert.

Zusammenfassend lassen sich folgende Schritte zur Erstellung eines DOM festhalten:

- Landnutzungspolygone festlegen

- Höhenversatz der Polygone einbinden

- Triangulation der Oberflächenrohdaten

- Umwandlung des TIN in einen Rasterdatensatz

- Fraktale bzw. generische Modulierung der Oberfläche

- Modulation der Waldränder

\section{Modifikation der Oberflächentextur}

Wie bereits erwähnt, weisen die verwendeten Luftbilder in Waldgebieten keine geschlossenen Waldoberflächen auf. Vielmehr ermöglichen sie den Blick bis zum Waldboden. Bei der Verwendung dieser Luftbilddaten als Textur für die Landschaftsdarstellung resultiert eine optisch nicht durchgehende Walddecke, obschon das DOM einen durchgehenden Wald aufweist. Um eine geschlossene Walddecke für unser DOM zu erhalten, ersetzen wir in den geschlossenen Waldgebieten das Luftbild durch eine generische Textur eines belaubten Waldes, die aus einem anderen Luftbild gewonnen wurde. Diese Modifikation des Luftbildes bringt zwar eine sichtbare Verbesserung der Visualisierung, führt aber zu einer Verfälschung der Bildinformation. Insbesondere korrelieren die Vegetationsstadien der bewaldeten resp. unbewaldeten Flächen nicht mehr.

Abb. 2 Links: schattiertes DGM; rechts: DOM mit angehobenen und modulierten Waldpartien. 


\section{Einfügen von Einzelbäumen}

Zur Darstellung von Einzelbäumen oder sehr kleinen Waldflächen ergibt die Anhebung des Geländes im DOM kein befriedigendes Ergebnis. Der Grund dafür ist, daß die Struktur eines Baumes nicht im 2,5-D-Raum modelliert werden kann. Wir modellieren daher Einzelbäume, indem mit Hilfe von teiltransparenten Texturen Baumprofile auf mehrere Ebenen projiziert und diese senkrecht zum Oberflächenmodell angeordnet werden (vgl. Abbildung 3, links). Die Plazierung erfolgt entweder an definierten Punkten oder mit einer vorgegebenen Dichte zufällig innerhalb einer Landnutzungsklasse. Die verschiedenen Polygone eines Baumes werden in der senkrechten Mittelachse gekreuzt. So wird aus jeder Blickrichtung eine räumliche Ausdehnung des Baumes erreicht. Die Polygone werden mit teiltransparenten Baumtexturen, die nach Möglichkeit im zu visualisierenden Gebiet photographisch aufgenommen wurden, überlagert.

Die Teiltransparenz der Textur bewirkt, daß in Teilen der Textur, die keine Bauminformation enthält, die dahinterliegenden Objekte sichtbar werden. Um eine Monotypie der Einzelbäume zu vermeiden, variieren wir einerseits die Größe der einzelnen Bäume, andererseits die horizontale Ausrichtung der Polygone.

Der Grad des Realismus der Einzelbäume wird erhöht, wenn in der Visualisierung die Schlagschatten der Baumpolygone z. B. mit einem Raytracer berechnet und dargestellt werden (FOLEY, VAN DAM 1994). Dabei muß bedacht werden, daß die verwendeten Luftbilder bereits Schlagschatten von Objekten enthalten. Deshalb müssen wir für die Generierung der Schlagschatten die gleiche Beleuchtungsrichtung wählen, wie sie zum Aufnahmezeitpunkt der Luftbilder herrschte.
Die Darstellung mit einzelnen Bäumen benutzen wir zusätzlich, um betrachternahe Waldflächen zu modellieren und so einen hohen Detaillierungsgrad im Vordergrund des Bildes zu erreichen (vgl. Abbildung 3, rechts). Die Modellierung der Wälder mit Einzelbäumen erhöht zwar die visuelle Realitätsnähe, hat aber einen höheren Rechenaufwand zur Folge.

\section{Datenvisualisierung}

\section{Verwendete Visualisierungssoftware}

Die von GRAF entwickelte Landschaftsvisualisierungssoftware EARTH ist ein vorzügliches Werkzeug zur Erzeugung perspektivischer Ansichten der vorliegenden Daten (GRAF 1995). Das Softwarepaket EARTH setzt sich zurzeit aus drei Modulen zusammen: dem Oberflächenvisualisierungs-, dem Atmosphären- und dem 3D-Objekt-Modul. Diese Module eröffnen vielfältige Möglichkeiten der Generierung von auf Fernerkundungs- und Geländedaten beruhenden Landschaftsansichten.

Vergleichbar mit dem menschlichen Sehsystem oder photographischen Kameras, erzeugt EARTH Bilder mit zentralperspektivischen Eigenschaften. Zur Erzeugung der Ansichten wird der Forward Mapping-Ansatz verwendet. Der Painter's Algorithmus (FOLEY, VAN DAM 1994) wird eingesetzt, um die Daten vom Objektraum in den Bildraum zu transferieren. Hierbei werden nahe liegende Punkte nach den entfernt liegenden Punkten berechnet.
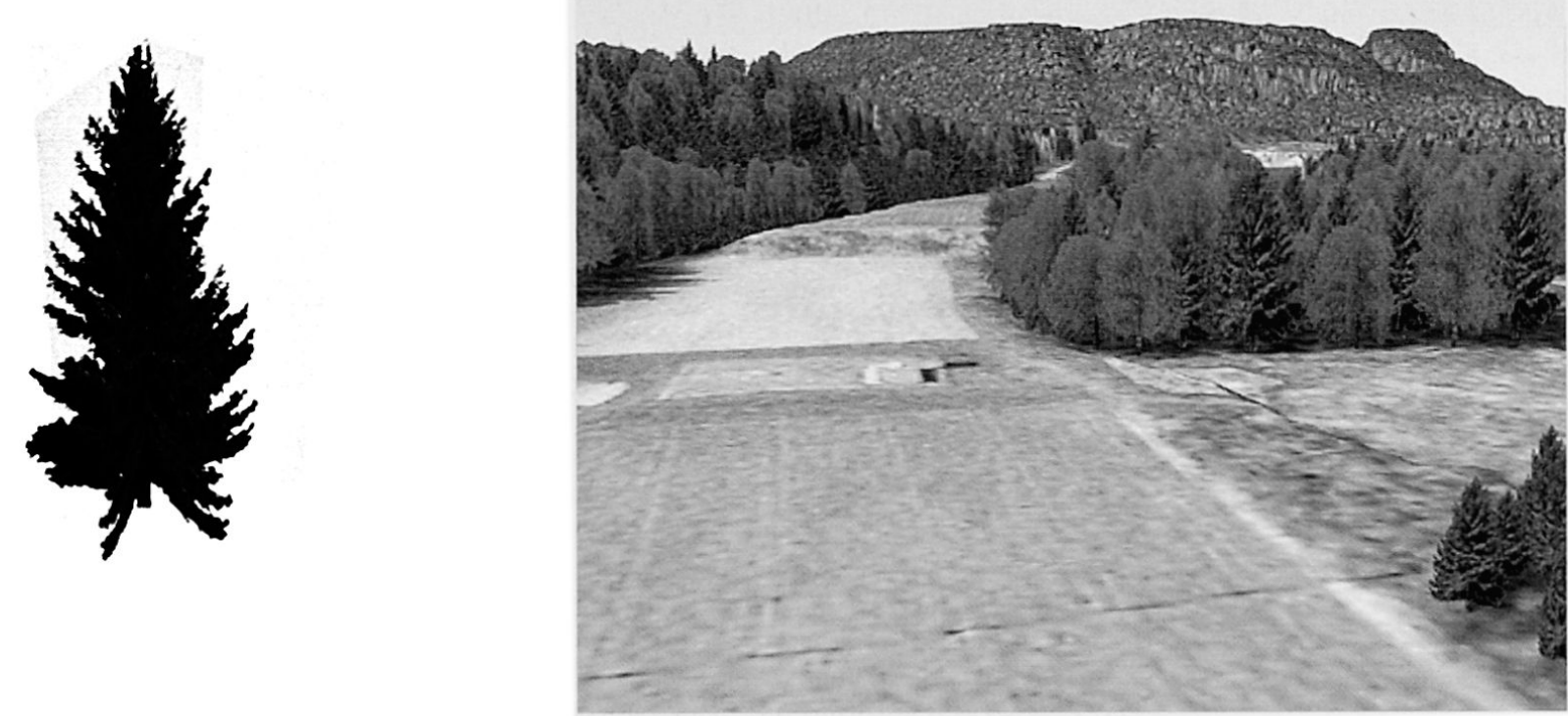

Abb. 3 Links: Beispiel eines Einzelbaumes aus drei gekreuzten Polygonen; rechts: Szene Region Töß mit Einzelbäumen im Vordergrund. 
Der Rechenaufwand wird durch Vorausscheidungstests reduziert, z. B. durch einen Test, ob sich die zu verwendenden Daten überhaupt im Blickfeld befinden.

Die sequentielle Verarbeitung der Daten erlaubt die Verwendung beliebig großer Datensätze. Im vorliegenden Fall ist die weitgehende Unabhängigkeit von der Hauptspeichergröße äußerst wichtig, müssen doch bei einer Gesamtvisualisierung rund 3,5 Gigabyte Bild- und 2,3 Gigabyte Geländedaten verarbeitet werden (vgl. Abbildung 4).

Ergänzend zur Funktionalität von EARTH, erlaubt die Verwendung eines Raytracers die Erzeugung von Objektschatten im Resultatbild. Bedingt durch die Architektur des verwendeten Raytracers, muß der Hauptspeicher die gesamte Szeneninformation aufnehmen können. Bei Verwendung von Daten der beschriebenen Auflösung erlaubt diese Einschränkung daher nur eine kleinräumige Visualisierung.

\section{Anwendungsgebiete}

Je nach Anwendung ist unterschiedliches Bildmaterial erforderlich. Großräumige Visualisierungen können beispielsweise mit TM-Szenen durchgeführt werden. Panoramische Ansichten aus der Vogelperspektive, Rundblicke von Aussichtspunkten oder Simulationen von Wetterphänomenen (z. B. Nebel mit wählbarer Obergrenze) sind sehr wirkungsvoll darstellbar.

Viele Anwendungen verlangen großmaßstäbliche Visualisierungen. Um diese naturnah darstellen zu können, bedarf es Daten hoher Auflösung. Durch geeignete Bearbeitung der Daten können nicht existierende Zustände, z. B. gemäß Szenarien, dargestellt werden.

Denkbare Anwendungsgebiete sind:

- Geowissenschaftliche Visualisierung räumlicher Daten: meteorologische Phänomene, geomorphologische Prozesse, Vegetationsdarstellung, Schadstoffausbreitung.

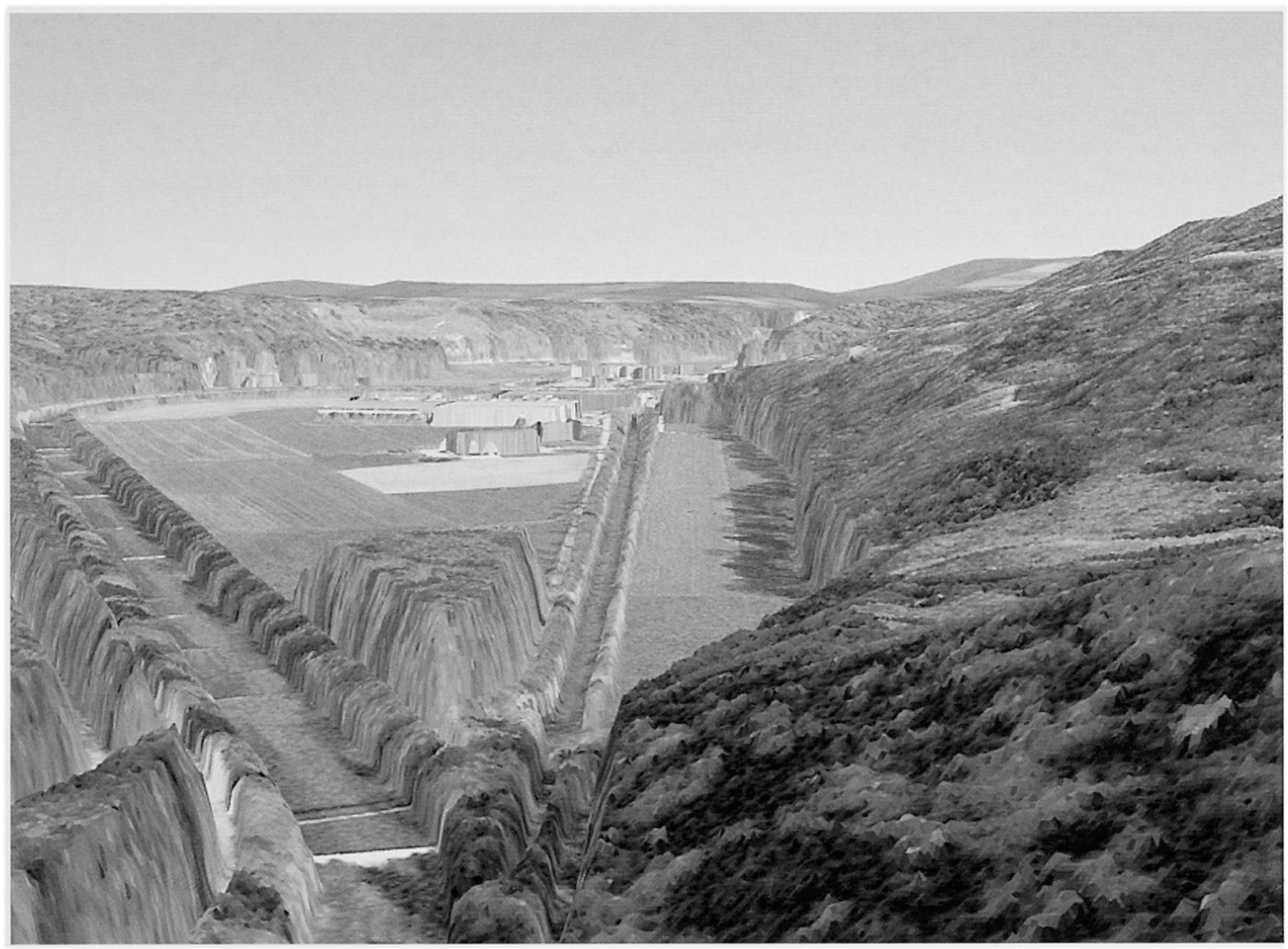

Abb. 4 Mit EARTH erzeugte Landschaftsansicht, Gebiet Töß bei Sennhof, Blickrichtung Nordost. 
- Planung: Raumplanung, Landschaftsarchitektur, Variantenstudium, Sichtbarkeitsanalyse, Schattenwurfanalyse.

- Projektpräsentation, -kommunikation.

- Touristisches Informationssystem: Nationalpark, interaktiver Stadtplan.

\section{Bewertung}

Mit den beschriebenen Visualisierungsmethoden können hochauflösende Luftbilder in einer perspektivischen Darstellung naturnah dargestellt werden. Sie ermöglichen eine beliebige Wahl des Betrachterstandpunktes und des Blickfeldes.

Die beschriebenen Methoden zur Modifikation des DGM bringen gute Resultate in Richtung eines erhöhten Realismus. Insbesonders bei einer hohen Datenauflösung werden durch ein unmodifiziertes DGM ungenügende Ergebnisse erzielt. Dank dem modifizierten DGM werden auch kleinräumige Sichtbarkeitsanalysen möglich. Eine Verbesserung könnte erreicht werden, wenn photogrammetrisch erfaßte Oberflächendaten zur Verfügung stünden, welche die Geländeoberfläche genauer repräsentieren als das von uns generierte DOM.

Die Visualisierung von Wäldern mit Hilfe eines DOM führt zu visuell guten Ergebnissen, wenn die verwendeten Luftbildtexturen eine geschlossene Laubdecke aufweisen. Bei einem boden- bzw. objektnahen Beobachterstandort erscheint es uns sinnvoll, die Vegetation als Einzelobjekte zu modellieren, um einen höheren Detaillierungsgrad zu erreichen. Andernfalls ist eine Modellierung mit Hilfe von Einzelbäumen denkbar. Der Qualitätsgewinn der räumlichen Auflösung von $10 \mathrm{~cm}$ gegenüber $50 \mathrm{~cm}$ steht in einem ungünstigen Verhältnis zu den großen Datenmengen, welche die Arbeit mit den hochauflösenden Datensätzen stark erschweren.

\section{Ausblick}

In weiteren Arbeitsschritten soll untersucht werden, auf welche Art die Gelände- und Bildinformation modifiziert werden kann, damit mögliche Szenarien zur Renaturierung des Flußlaufes visuell dargestellt werden können. Diese können einerseits zur Unterstützung von Planern und Entscheidungsträgern, andererseits als Informationsmaterial verwendet werden.

Weitere Forschungsarbeiten werden in Richtung der interaktiven Simulation der Daten angesetzt. Dabei soll dem Betrachter ermöglicht werden, sich im Untersuchungsgebiet frei zu bewegen. Dafür bedarf es der Einbindung der Daten in eine Datenstruktur, welche die Darstellung in Echtzeit gerechneter Bilder erlaubt.

\section{Dank und Copyrights}

Wir danken Jens Piesbergen, Hilko Hoffmann und Philippe Meuret für ihre Anregungen und Hinweise. Die Besitzrechte an den verwendeten Luftbilddaten und hochauflösenden Geländemodelldaten halten das AGW des Kantons Zürich und das Institut für Umwelt-Risiko-Management, Interkantonales Technikum Rapperswil. Die Satellitenbilddaten stammen von Eurimage.

\section{Literatur}

FOLEY, J. D., VAN DAM, A. (1994): Grundlagen der Computergraphik. Addison-Wesley, Bonn.

GRAF, K. CH., SUTER, M., HAGGER, J., NÜESCH, D. (1994): Computer Graphics and Remote Sensing - a Synthesis for Environmental Planning and Civil Engineering. Proceedings of Eurographics '94, Computer Graphics Forum, Vol. 13, Nr. 3, Oslo, 13-22.

GRAF, K. CH. (1995): Realistic Landscape Rendering using Remote Sensing Images, Digital Terrain Models and 3D Objects. Dissertation Universität Zürich, Remote Sensing Series, Vol. 25.

SUTER, M. (1992): Digitales Mosaik der Schweiz aus Landsat-Thematic-Mapper-Satellitendaten. Diplomarbeit, Geographisches Institut der Universität Zürich. 